\title{
¿Qué hacer con la ciencia y la tecnología en la recuperación del país? Veinte propuestas para el futuro gobierno
}

Resumen: Las elecciones presidenciales del presente año abren una posibilidad de cambio de rumbo en políticas de Estado. El enfoque neoliberal del actual gobierno nacional y las medidas que ha implementado han producido un enorme retroceso social, político y económico del país y, en particular, en el sector de la Ciencia y la Tecnología. Frente a la posibilidad de una eventual recuperación de un proyecto de país centrado en la producción, el trabajo, la soberanía y la inclusión, quienes integramos la Cátedra Libre "Ciencia, Política y Sociedad" presentamos veinte propuestas en CyT para el futuro gobierno. Por un lado, diez medidas de emergencia para enfrentar la crisis que atraviesa el complejo científico tecnológico actual. Por otro lado, diez propuestas de mediano y largo plazo para que el sector de Ciencia y Tecnología pueda contribuir activamente a la recuperación del país.

Palabras clave: Argentina; política científica; pensamiento latinoamericano; desarrollo nacional; soberanía; ciencia y tecnología

\section{What to do with science and technology in the country's recovery? Twenty proposals for the future government}

Abstract: The elections that will take place this year represent a chance of redirection in state policies. The neoliberal approach of the current national government and the measures it has implemented have produced a huge social, political and economic retreat of the country and, particularly in science and technology. Faced with the possibility of recovering a country project focused on production, work, sovereignty and inclusion, those who are part of the Cátedra Libre "Ciencia, Politica y Sociedad" present our twenty proposals in S\&T for the next government. On the one hand, ten emergency measures addressed to deal with the crisis already affecting the scientific and technogical system. On the other hand, ten medium and long-term proposals to engage the Science and Technology sector in the country's rebuilding after neoliberalism.

Keywords: Argentina; scientific policy; Latin American thought; national development; sovereignty; science and technology eignty; science and technology 


\section{O que fazer com a ciência e tecnologia na recuperação do país? Vinte propostas para o futuro governo}

Resumo: As eleições presidenciais deste ano abrem a possibilidade de uma mudança de rumo nas políticas de Estado. O enfoque neoliberal do atual governo nacional e as medidas que implementou produziram um enorme retrocesso social, político e econômico no país e, em particular, no setor de ciência e tecnologia. Diante de uma eventual recuperação de um projeto de país focado na produção, no trabalho, na soberania e na inclusão, nós que integramos a Cátedra Livre "Ciência, Política e Sociedade" apresentamos vinte propostas em CyT para o futuro governo. Por um lado, dez medidas emergenciais para enfrentar a crise que atravessa o atual complexo científico-tecnológico. Por outro lado, dez propostas de médio e longo prazo para que o setor de Ciência e Tecnologia possa contribuir ativamente para a recuperação do país.

Palavras-chave: Argentina; política científica; Pensamento latino-americano; desenvolvimento nacional; soberania; Ciência e Tecnologia

\section{Introducción}

La posible recuperación en octubre de 2019 de un proyecto de país centrado en la producción, el trabajo, la soberanía y la inclusión requiere la elaboración de políticas públicas para que el complejo científico tecnológico argentino pueda jugar un rol activo en este proceso.

Argentina cuenta actualmente con un conjunto numeroso de instituciones que realizan investigación científica y desarrollos tecnológicos (universidades nacionales, CONICET, CONAE, CNEA, INTI, INTA, etc.). Este conglomerado no tiene los rasgos de un Sistema Nacional de Ciencia y Tecnología sino más bien de un Complejo Científico-Tecnológico, ya que, como es generalmente reconocido, carece de coordinación interna y se encuentra mayormente desarticulado de aquellos sectores con los que debería interactuar (aparato productivo, sistemas de salud y educación, áreas de energía y recursos naturales, defensa, etc.). Esta fragmentación institucional e intersectorial es propia de los países dependientes y se configura en virtud de la ausencia de un proyecto nacional nucleador.

Por esta razón creemos que se hace necesario construir un sistema científico-tecnológico articulado a un Proyecto Nacional basado en el desarrollo, la soberanía y la inclusión social. Esto significa, en el mediano y largo plazo, construir el nexo entre proyecto nacional, matriz organizacional y productiva, sistema de CyT y sociedad. Proceso que supone un amplio acuerdo político entre los distintos sectores y actores sociales que lo impulsen. Esta construcción implica una transversalidad que abarca, entre otros, los planos económico, social, cultural, educativo, político, ambiental y de recursos naturales. Por tanto, la constitución de este sistema y la elaboración de una política científico-tecnológica requieren de diagnósticos y propuestas que trascienden al sector. Es un problema político que debe involucrar a diversos actores.

En este marco se debería elaborar, para el mediano y largo plazo, un plan estratégico de desarrollo nacional, con políticas explícitas en grandes áreas tales como hábitat, salud, educación, energía, 
recursos naturales, producciones regionales y medio ambiente. Mientras que, en el corto plazo, se hace necesario enfrentar la crisis que atraviesa el complejo científico tecnológico actual, a fin de evitar que continúe su desmantelamiento y destrucción. Esto requiere tomar medidas de emergencia que permitan revertir la situación y poner a nuestras instituciones científico-tecnológicas en condiciones de participar activamente en la reconstrucción nacional. Los horizontes estratégicos generales, en esta etapa, pueden ser pensados en términos de lograr soberanía económica, alimentaria, educativa, cultural, industrial, energética y tecnológica. Estas medidas deben tomarse desde una perspectiva federal que reconozca las distintas realidades regionales y provinciales potenciando las estrategias que generan localmente políticas públicas para la inclusión, la disminución de la desigualdad y la distribución de la riqueza.

Basándonos en estos conceptos, desde la Cátedra Libre de la Universidad Nacional de la Plata Ciencia, Política y Sociedad, presentamos a continuación veinte propuestas para que el sector de Ciencia y Tecnología pueda contribuir activamente a la recuperación del país. De las cuales diez son medidas de emergencia para su ejecución en forma inmediata. Las otras diez son propuestas para que, en un mediano y largo plazo, podamos avanzar en la constitución de un sistema científico y tecnológico nacional basado en la utilización del conocimiento para el desarrollo, la soberanía y la inclusión social.

\section{Propuestas para enfrentar la emergencia y para su implementación inmediata}

1. Recuperar el nivel ministerial de la actual Secretaría de Ciencia, Tecnología e Innovación Productiva y activar instancias de coordinación interministerial e interinstitucional (por ejemplo, GACTEC, CICyT y COFECyT) para articular acciones inmediatas en todos los organismos de ciencia y tecnología, que permitan movilizar los recursos y capacidades en función de resolver necesidades urgentes en materia de salud, energía, comunicaciones, hábitat, producción, servicios, medio ambiente y defensa, entre otras prioridades.

2. Reincorporar al personal científico/técnico/administrativo injustamente despedido de todos los organismos de ciencia y tecnología. Junto con la revisión y recuperación de las metas estratégicas en cada organismo, reabrir la incorporación de personal en aquellas instituciones que hoy tienen su planta congelada.

3. Aumentar la participación de la función Ciencia y Técnica en el presupuesto nacional para compensar la caída que sufrió en los últimos años.

4. Aumentar los salarios de investigadores/as y personal técnico y administrativo y el monto de las becas en todos los organismos de ciencia y tecnología para recuperar el 30-35\% perdido durante estos últimos años. Abrir las negociaciones y el debate sobre la concreción de un Convenio Colectivo Único para todos los organismos científico-tecnológicos (CONICET, INTA, INTI, CNEA, CONAE, CICPBA, UUNN, etc.), en el que se eliminen todas las formas de precarización laboral. Esta "homogeneización" contribuiría, además, a la coordinación y movilidad entre instituciones.

5. En CONICET y otros organismos e instancias ejecutoras de las políticas de CyT: 
a) Transformar el sistema de becas doctorales y postdoctorales en contratos a término con plenos derechos laborales que incluyan aportes, vacaciones, licencias y antigüedad.

b) Promover la igualdad de género en los concursos, comisiones evaluadoras, puestos de gestión, etc. Adecuar las convocatorias de ingreso a la Carrera del Investigador Científico, del Personal de Apoyo y las presentaciones para financiamiento de proyectos, para que las postulantes que han sido madres en la etapa previa a las presentaciones tengan iguales oportunidades. Incorporar el derecho a licencia por paternidad.

c) Inclusión en el régimen de jubilación previsto en la Ley N²2.929 al personal de la Carrera de Personal de Apoyo de CONICET.

d) Revisar los mecanismos y criterios de evaluación en las diferentes instancias. En particular, atendiendo a las especificidades de las actividades tecnológicas, por un lado, y de las Ciencias Sociales y Humanidades, por el otro.

e) Adoptar una política soberana de publicaciones que, entre otras cosas, discuta qué se publica y qué no, dónde publicar y cómo publicarlo; además, optimizar los recursos que el Estado destina al pago para el acceso a revistas científicas de grandes editoriales privadas y favorecer las publicaciones de Acceso Abierto.

f) Recuperar el programa RAÍCES de repatriación de científico/as en el exterior.

6. Retomar los objetivos estratégicos del sector nuclear: finalización del reactor de potencia media CAREM, reactivación del acuerdo con China orientado a la compra de dos reactores (uno de ellos un reactor CANDU, que supone el $75 \%$ de participación de industria nacional), recuperación de la cadena de valor del ciclo del combustible nuclear y producción de agua pesada.

7. Revisar la política aeroespacial y satelital volviendo a poner en vigencia la Ley $N^{\circ} 27.208 / 15$ de promoción de la industria satelital. Recuperar el rol de ARSAT en el desarrollo de infraestructura para las telecomunicaciones (Red Federal de Fibra Óptica, etc.) y reactivar la fabricación del ArSat-3. Recuperación del Plan Espacial orientado a satélites de observación y geoestacionarios y el lanzador satelital Tronador. Fortalecer el desarrollo nacional de radares tanto para uso doméstico como para eventual exportación.

8. Reactivar una política de producción pública de medicamentos a través del fortalecimiento de la Agencia Nacional de Laboratorios Públicos (ANLAP) y la Administración Nacional de Laboratorios e Institutos de Salud (ANLIS) con énfasis en la promoción de proyectos destinados a resolver necesidades públicas esenciales en materia de salud.

9. Reformular la actual política de importación de tecnología "llave en mano" en el área de energías renovables, integrándolas a una política energética que apunte a la máxima incorporación de ciencia, tecnología y componentes nacionales, en un sector en que nuestro país tiene capacidad para ser competitivo.

10. Reactivación mediante la ejecución del financiamiento aprobado oportunamente por Ley № 27.167 del proyecto estratégico Pampa Azul sobre la base de que la presencia científica permanente en nues- 
| Ciencia, Tecnología y Política | Año 2 | №2 | Enero-Junio 2019 | ISSN 2618-3188 | www.revistas.unlp.edu.ar/CTyP |

tros territorios marinos es un pilar fundamental para fortalecer la soberanía nacional.

\section{Propuestas de mediano y largo plazo}

1. Hacia una política de Estado en CyT. Convocar a una instancia nacional y a instancias regionales y municipales para la elaboración de las políticas públicas de Ciencia y Tecnología que deberían impulsarse y desarrollarse en el país. En estas instancias deberían participar PyMEs, cooperativas, cámaras empresariales, sindicatos, movimientos y organizaciones sociales y de la economía popular, empresas públicas (INVAP, YTEC, Fabricaciones Militares, ARSAT, Nucleoeléctrica, etc.), sectores del Estado vinculados con las áreas de salud, educación, medio ambiente, defensa, etc., y partidos políticos. Por otro lado, deberían participar actores vinculados directamente con la producción de conocimientos, con la ejecución de las políticas definidas para el sector y con la formación de los recursos humanos involucrados y requeridos para llevarlas adelante (instituciones científicas, universidades, sistema educativo, gestores, asociaciones científicas y profesionales, entre otros). La articulación intersectorial, interdisciplinaria y política de estos actores debería elaborar diagnósticos de necesidades, definir prioridades para el sector, seleccionar proyectos a desarrollar y generar estudios prospectivos para dar respuesta a los problemas planteados. El resultado de estas acciones debería constituirse en un Plan Nacional de Ciencia y Tecnología (PNCyT). Este plan debería contemplar, además, instancias de evaluación que aseguren su cumplimiento (observatorios, comisiones intersectoriales, instancias parlamentarias, etc). Los ejes rectores de este PNCyT deberían estar basados en la Soberanía Científico-Tecnológica y el Desarrollo Nacional. Esto es: poner el conocimiento, los recursos y el complejo CyT del país en proyectos liderados por el Estado destinados a resolver necesidades nacionales y regionales de carácter estratégico, social, económico o ambiental, en el marco de un proyecto nacional de desarrollo, soberanía e inclusión social.

2. Un nuevo modelo de MinCyT. Diseñar un Ministerio de Ciencia y Tecnología encargado principalmente de la ejecución de este Plan Nacional de Ciencia y Tecnología que debería contar, por un lado, con el presupuesto y los recursos humanos para llevarlo adelante y, por otro lado, que debería ocuparse de la planificación, agenda, diseño de instrumentos, definición de etapas, evaluación, etc. Integrar a este Ministerio a aquellos organismos e instituciones del complejo científico-tecnológico que se consideren necesarios para avanzar en la articulación de un sistema de CyT. En el marco del PNCyT este Ministerio junto con Jefatura de Gabinete, CONICET, Universidades Nacionales, organismos descentralizados de CyT y organismos públicos (Nacionales, Provinciales y Municipales), debería realizar una planificación de corto y mediano plazo que permita la inserción de recursos humanos en cada organismo y en otros sectores. Por otra parte, desde esta instancia se debería iniciar un proceso de acumulación de capacidades estatales para el diagnóstico, gestión y prospectiva en el área de políticas tecnológicas.

3. Una nueva Ley Federal de CyT. Promover una Ley de Ciencia y Tecnología que, entre otros aspectos, establezca el rol del sistema científico-tecnológico como consultor estratégico del Estado. A su vez debería garantizar su financiamiento ininterrumpido y a largo plazo. Esta ley debería incluir explícitamente aspectos referidos a la federalización del sector que permitan un desarrollo equilibrado de las actividades de CyT en todo el país, mecanismos para el impulso de proyectos generados por 
las comunidades locales y la búsqueda de soluciones a los problemas regionales. También debería incluir iniciativas que estimulen la radicación de investigadores en las provincias con menos densidad de recursos humanos en I+D, dotándolas de la infraestructura necesaria (edificios, laboratorios y equipamiento).

4. Hacia un Estado emprendedor con políticas sectoriales en CyT. Entendiendo que el Estado es el real impulsor de los grandes desarrollos productivos, en especial en países dependientes como el nuestro, proponemos:

a) Impulsar y consolidar el crecimiento de aquellas áreas estratégicas que involucran recursos y capacidades CyT y que en diferente grado ya se han ido configurando en el país. Entre ellas la energía nuclear, el desarrollo aeroespacial, aeronáutico, naval y ferroviario, la producción pública de medicamentos, etc.

b) Promover la creación de empresas estatales (públicas o mixtas, según el caso) en áreas en las que se cuenta con capacidades CyT y activos estratégicos como, por ejemplo, litio, biotecnología, etc.

c) Elaborar estrategias CyT para el agregado de valor en industrias extractivas como la pesca y la minería, el gas y el petróleo.

d) Reformular el papel del Estado y la CyT para generar eslabonamientos locales virtuosos y agregación nacional de valor en ciertas áreas de la actividad privada (software, siderurgia, cemento, etc.).

5. La contribución de la СуT a la redistribución de la renta agraria. El resultado de la disputa por la renta agraria concentrada define en buena medida las posibilidades de un modelo de desarrollo con sentido nacional. Es la principal fuente de ingresos para el país y un sector en que tenemos ventajas comparativas (excelentes tierras y climas), acumulación de conocimientos (agronómicos, biotecnológicos, etc.) y capacidades científico-tecnológicas para el procesamiento industrial. El modelo de agronegocios imperante se desarrolla según cadenas globales de valor y el Estado apenas ha sido capaz de captar un parte de la renta agraria mediante impuestos a la exportación. Creemos que la CyT es parte fundamental de una estrategia soberana en relación al sector agrario. Por eso proponemos:

a) Establecer una estrategia específica para el fortalecimiento mediante la CyT de las industrias de maquinarias, farmacología agrícola y procesamiento de materias primas de origen agropecuario, entendiendo que Argentina puede lograr desarrollos de vanguardia en esas áreas, sustituir importaciones y generar divisas mediante exportación con valor agregado.

b) Implementar una política soberana en materia de semillas, genética animal y vegetal, mediante la creación de un Banco Público de Germoplasma y una reforma a la Ley de Semillas que permita proteger el conocimiento y la producción nacionales, evitando la cartelización de los proveedores y comercializadores.

c) Revisar las estrategias de patentamiento y comercialización de los desarrollos agrobiotecnológicos realizados en organismos públicos, para que el Estado pueda recaudar efectivamente los beneficios derivados de la innovación.

d) Proteger y fortalecer a la pequeña y mediana producción familiar mediante la capacitación técnica, 
el desarrollo y provisión de tecnologías acordes a su escala y el agregado de valor en origen.

6. СуT para el uso sustentable de recursos naturales y monitoreo ambiental. Impulsar la creación de un Observatorio Nacional Ambiental destinado al estudio científico, interdisciplinario, público, transparente y confiable sobre la sustentabilidad socio-ambiental de las diferentes cadenas productivas. Su principal finalidad debería ser el monitoreo ambiental de la explotación de los recursos naturales. El trabajo de este organismo debería garantizar mecanismos de participación ciudadana y fortalecer el trabajo de las agencias provinciales y organismos de regulación.

7. Fortalecimiento de la economía popular mediante la CyT. Nuestro país tiene un tercio de su población en condiciones de exclusión social y millones de trabajadores precarizados que desarroIlan lo que se denomina economía popular. Se trata de una actividad económica con baja intensidad tecnológica en las áreas de producción de alimentos, la recuperación de residuos urbanos, la manufactura textil, construcción y reparación de viviendas, venta ambulante, etc. Proponemos que el complejo CyT pueda brindar soluciones técnicas, o complementar respuestas socio-técnicas en curso, que permitan a los actores más débiles de las cadenas de valor mejorar la condiciones de trabajo, así como apropiarse de una mayor parte de los beneficios (tecnologías para el reciclado, ferias urbanas de comercio justo, herramientas de logística y gestión, conocimientos agrícolas, etc.). Experiencias realizadas en el INTI e INTA son antecedentes en ese sentido.

8. Política soberana en relación a Derechos de Propiedad Intelectual. Revisión de los términos del Acuerdo sobre los Aspectos de los Derechos de Propiedad Intelectual relacionados con el Comercio (ADPIC) desde una perspectiva estratégica nacional (lo que supone rediscutir la Ley de Patentes, el Régimen Legal de la Propiedad Intelectual, etc.). La actual configuración de los Derechos de Propiedad Intelectual es una limitación para el desarrollo de países dependientes como el nuestro. No se debería incentivar ingenuamente a científicos/as y empresario/as a patentar, sino hacerlo en el marco de estrategias que permitan al país la captación de los beneficios derivados de la innovación. Impulsar una política de innovación que combine diversas estrategias en el sentido de "mix tecnológico" propuesto por Jorge Sábato.

9. Generar un cambio cultural en el sector de CyT. Es necesario modificar mecanismos de reproducción que el sector de CyT argentino ha generado a lo largo de su evolución y que son en buena medida una copia, simplificada y descontextualizada, de los utilizados en los países centrales. Se hace necesario contraponer a la formación de recursos humanos tecnocrática, meritocrática y cientificista que hoy impera, una formación crítica y comprometida con la utilización del conocimiento en la resolución de los problemas del país. Para ello proponemos la inclusión de cursos, seminarios y capacitaciones en el grado, posgrado y ejercicio profesional que contengan, entre otros contenidos, una reflexión sobre la responsabilidad social de la actividad CyT, las implicancias éticas y políticas de las investigaciones y desarrollos y la importancia de adoptar una mirada crítica sobre el quehacer científico y tecnológico.

10. Repensar el papel de las Universidades en el complejo Сут. Las Universidades Nacionales tienen diversas funciones. Aquí nos interesa destacar su aporte a un posible Sistema Nacional de CyT, 
tanto en relación a la investigación científica que en ellas se realiza como a su rol de formadoras de los recursos humanos de máximo nivel que las otras instituciones científicas y tecnológicas absorben. Es necesario una nueva Ley Nacional de Educación Superior que integre la evolución, orientación y expansión de las Universidades a una estrategia nacional de desarrollo. La ausencia de una planificación estratégica en el crecimiento de estas instituciones ha conducido en muchos casos a una duplicación de esfuerzos, con el costo que supone para el erario público. En el mismo sentido, es preciso revisar las lógicas neoliberales de investigación vigentes desde las reformas de los ‘90. En particular, el Programa de Incentivos a Docentes-Investigadores. Por último, destaquemos que el despliegue territorial de las universidades y su trayectoria en la extensión las vuelven el actor clave en la vinculación entre demandas sociales y complejo de CyT, uno de los desafíos fundamentales en la construcción de un Sistema Nacional de Ciencia y Tecnología.

La Plata, 31 de marzo de 2019

http://blogs.un/p.edu.ar/catedracps/

https://revistas.un/p.edu.ar/CTyP 